\title{
Publisher Correction: Functional polymers in nonpolar solvents induced by dissociation of macromolecular complexes
}

\author{
Kazuki Sada ${ }^{1}$
}

Published online: 5 June 2018

(c) The Society of Polymer Science, Japan 2018

Correction to: Polymer Journal 50, 285-299 (2018) https://doi.org/10.1038/s41428-017-0016-4, published online 07 February 2018

In the original version of this article, owing to an error during the production process, the author biography was unfortunately missed. The correct author biography is given below.

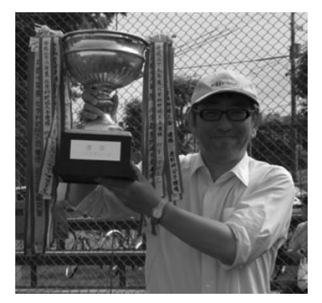

Kazuki Sada was born in 1963 in Nagoya (Aichi), Japan. He received his M.A. in 1987 from Kyoto University under the direction of the late Professor Iwao Tabushi, and his Ph.D. degree in 1991 from the same university under the guidance of Professor Takeo Saegusa, focusing on the synthesis of functional hydrogels based on poly(2-alkyl-2oxazoline)s. In 1990, he started an academic career at Department of Applied Chemistry, Faculty of Engineering, Gifu University, as an assistant professor in the group of Professor Mikiji Miyata. In 1995, he moved to Osaka University along Professor Miyata's research group. In 2002, he joined Professor Seiji Shinkai's group as an associate professor at Department of Chemistry and Biochemistry, Graduate School of Engineering, Kyushu University. Since 2010, he is a full professor at Department of Chemistry, Faculty of Science, Hokkaido University. Honors: "Prizes for Science and Technology, Research Category", The Commendation for Science and Technology by the Minister of Education, Culture, Sports, Science and Technology, Japan in 2008 and "31st Chemical Society of Japan Award for Creative Work" in 2014, and "The Award of the Society of Polymer Science, Japan (2016)".
Kazuki Sada

sadatcm@sci.hokudai.ac.jp

1 Department of Chemistry, Faculty of Science, Hokkaido University, Sapporo, Hokkaido 060-0810, Japan 